\title{
Regularized Fractional Power Parameters for Image Denoising Based on Convex Solution of Fractional Heat Equation
}

\author{
Hamid A. Jalab \\ Faculty of Computer Science and Information Technology, Multimedia Unit, University of Malaya, \\ 50603 Kuala Lumpur, Malaysia \\ Correspondence should be addressed to Hamid A. Jalab; hamidjalab@um.edu.my \\ Received 11 February 2014; Revised 8 April 2014; Accepted 12 May 2014; Published 25 May 2014 \\ Academic Editor: Dumitru Baleanu \\ Copyright (c) 2014 Hamid A. Jalab. This is an open access article distributed under the Creative Commons Attribution License, \\ which permits unrestricted use, distribution, and reproduction in any medium, provided the original work is properly cited. \\ The interest in using fractional mask operators based on fractional calculus operators has grown for image denoising. Denoising \\ is one of the most fundamental image restoration problems in computer vision and image processing. This paper proposes an \\ image denoising algorithm based on convex solution of fractional heat equation with regularized fractional power parameters. The \\ performances of the proposed algorithms were evaluated by computing the PSNR, using different types of images. Experiments \\ according to visual perception and the peak signal to noise ratio values show that the improvements in the denoising process are \\ competent with the standard Gaussian filter and Wiener filter.
}

\section{Introduction}

Fractional calculus is an area of mathematical analysis; it generalized the ideas of integer order differentiation and $n$ fold integration. The fractional derivatives have a history of more than three centuries. However, the fractional calculus was seen as a pure mathematical branch without real life applications for a certain period of time until few decades ago when it was found that the fractional calculus was not only useful but also powerful [1-4]. During the last decade, many mathematicians have much different contribution to all fields of science. The advantages of fractional differentiations become apparent in signal processing, modeling, and control as well as in the description of properties of liquids, gases, and rocks and in many other applications $[1,5-7]$.

The fractional calculus in the field of image processing has received considerable attention in various image processing applications such as texture enhancement and image denoising [8-16]. Image denoising refers to the process of restoring a digital image that has been contaminated by any type of noise. It is an important preprocessing task for segmentation, feature extraction, texture analysis, and other image processing applications. Noise, arising from avariety of sources, is inherent to all electronic image sensors and the electronic components in the image environment. In additive white Gaussian noise, all the image pixels deviate from their original values following the bell-shaped curve distribution (Gaussian curve).

Fractional integral is extensively used in many image denoising algorithms. References $[10,12]$ have proposed a fractional integral denoising algorithm and the implementation of fractional integral filter using fractional integral masks on eight directions, based on fractional calculus RiemannLiouville definition. The results in [14] showed that the fractional integral mask in the Grünwald-Letnikov sense achieved fine-tuning of image denoising. It has been proved in [11] that the generalized Srivastava-Owa fractional integral operator algorithm not only enhances the quality of filtered image but also reserves the textures and edges present in the image.

The class of fractional heat equation is studied widely in the recent years. It included various types of special equations such as heat conduction equation, fractal heat transfer, fractional subdiffusion equation, and fractional heat equation of porous medium [16-18].

The effectiveness of fractional heat equation makes it an attractive work for many researchers [19-21]. All these works used time fractional differential equation; however, no previous works have been done using time-space fractional 
heat equation for image denoising. The partial heat differential equations have played a significant role in image enhancements. Heat equation is called isotropic diffusion process, which is extremely used for smoothing entire images uniformly like a Gaussian filter.

In this paper, we extend our previous work from [22] and utilize the concept of the fractional heat diffusion (FHD) for image denoising, which is the main contribution of this work. The denoising performance based on convex solution of fractional heat equation is measured by employing experiments according to standard of visual perception and by using peak signal to noise ratio (PSNR).

This paper is organized as follows. In Section 2, we introduce the concept of convex solution of the fractional heat diffusion. In Section 3, construction of fractional masks is presented. The experimental results and comparison with other works are shown in Sections 4 and 5, respectively. Finally, conclusion is presented in Section 6.

\section{Convex Solution of the Fractional Heat Diffusion}

In this section, we proceed to derive a mathematical model that utilizes the convex solution of the fractional heat diffusion for image denoising. In our previous work [22], we studied the maximal solution of time-space fractional heat equation in a complex domain:

$$
\begin{aligned}
& D^{\alpha} u(t, z)=D_{z}^{\gamma} u(t, z), \quad u(0, z)=0, \\
& (t \in[0, T], z \in U, \alpha \in(0,1), \gamma \in[1,2)),
\end{aligned}
$$

and $u: J \times U \rightarrow U$. The source of the heat is in the unit disk in neighborhood of the origin.

Lemma 1 (see [22]). Let $u(t, z)$ be convex function in the unit disk for all $t \in J$. Then (1) has a maximal solution $u(t, z)$ in the domain $D=J \times U$ of the form

$$
\begin{aligned}
u(t, z)= & \frac{r^{-\gamma}}{\Gamma(1-\gamma) \Gamma(1+\alpha)} \\
& \times F\left((2)_{n},(1)_{n},(1)_{n} ;(1-\gamma)_{n},(1+\alpha)_{n} ; r t^{\alpha}\right), \\
& \quad(0<|z|=r<1 ; 0<\gamma<1,0<\alpha<1) .
\end{aligned}
$$

\section{Construction of FHD Masks}

Using the fractional operators defined in Section 2, we proceed to construct FHD masks, which is the main contribution of this work. Our aim is to utilize (2) to formulate our fractional masks. The nonzero values of corresponding terms in formula (2) are

$$
\begin{aligned}
\psi_{0}= & \frac{r^{-\gamma}}{\Gamma(1-\gamma) \Gamma(1+\alpha)} \\
& \times F\left((2)_{0},(1)_{0},(1)_{0} ;(1-\gamma)_{0},(1+\alpha)_{0} ; r t^{\alpha}\right), \\
\psi_{1}= & \frac{r^{-\gamma}}{\Gamma(1-\gamma) \Gamma(1+\alpha)} \\
& \times F\left((2)_{1},(1)_{1},(1)_{1} ;(1-\gamma)_{1},(1+\alpha)_{1} ; r t^{\alpha}\right), \\
\psi_{2}= & \frac{r^{-\gamma}}{\Gamma(1-\gamma) \Gamma(1+\alpha)} \\
& \times F\left((2)_{2},(1)_{2},(1)_{2} ;(1-\gamma)_{2},(1+\alpha)_{2} ; r t^{\alpha}\right), \\
\vdots & \quad \times F\left((2)_{n},(1)_{n},(1)_{n} ;(1-\gamma)_{n},(1+\alpha)_{n} ; r t^{\alpha}\right) . \\
\psi_{n-1}= & \frac{r^{-\gamma}}{\Gamma(1-\gamma) \Gamma(1+\alpha)}
\end{aligned}
$$

The coefficients of two-dimension FHD masks can be obtained as an infinite series using (3) in eight directions: $0^{\circ}, 45^{\circ}, 90^{\circ}, 135^{\circ}, 180^{\circ}, 225^{\circ}, 270^{\circ}$, and $315^{\circ}$ (Figure 1). The technical viewpoint of this work is based on splitting the whole corrupted image to nonoverlapping blocks. The filter scheme of FHD approach is based on direct processing for discrete pixels, by moving the FHD mask window pixel by pixel, which will remove the noise from the corrupted images, which is the main aim of this study. The final new filtered image based on FHD can be obtained by the summation of all eight convolution results of the magnitudes for each filter. The complexity of the denoising algorithms depends mainly on the masks size, and to accomplish lower complexity, the size of the masks should be small.

\section{Experiments and Discussion}

In this section, we demonstrate the denoising performance of FHD masks. Performance tests for the proposed algorithm were implemented using MATLAB R2013a and Windows 7. The testing images used are as follows:

(i) grayscale images "Boat," "Lena," and “Taj”;

(ii) color images "Pepper" and "House."

The images are degraded with Gaussian noise with different standard deviation $\sigma$ values $(15,20$, and 25). The values of the fractional powers of the proposed masks are defined with the range of $0<\alpha \leq 1$ and $\gamma<1$. The performance of FHD algorithm, Gaussian filter, and Wiener filter has been evaluated by using PSNR, which is defined via the mean squared error (MSE) [11].

There are two fractional power parameters in our algorithm $\alpha$ and $\gamma$. In Figure 2, we first display the behavior of PSNR for the values of $\alpha$, ranging from 0.1 to 1 , at $\gamma=$ 


\begin{tabular}{|c|c|c|c|c|}
\hline 0 & 0 & 0 & 0 & 0 \\
\hline 0 & 0 & 0 & 0 & 0 \\
\hline$\Psi_{n}$ & $\cdots$ & $\Psi_{2}$ & $\Psi_{1}$ & $\Psi_{0}$ \\
\hline 0 & 0 & 0 & 0 & 0 \\
\hline 0 & 0 & 0 & 0 & 0 \\
\hline
\end{tabular}

(a)

\begin{tabular}{|c|c|c|c|c|}
\hline 0 & 0 & 0 & 0 & 0 \\
\hline 0 & 0 & 0 & 0 & 0 \\
\hline$\Psi_{0}$ & $\Psi_{1}$ & $\Psi_{2}$ & $\cdots$ & $\Psi_{n}$ \\
\hline 0 & 0 & 0 & 0 & 0 \\
\hline 0 & 0 & 0 & 0 & 0 \\
\hline
\end{tabular}

(c)

\begin{tabular}{|c|c|c|c|c|}
\hline 0 & 0 & 0 & 0 & $\Psi_{n}$ \\
\hline 0 & 0 & 0 & $\cdots$ & 0 \\
\hline 0 & 0 & $\Psi_{2}$ & 0 & 0 \\
\hline 0 & $\Psi_{1}$ & 0 & 0 & 0 \\
\hline$\Psi_{0}$ & 0 & 0 & 0 & 0 \\
\hline
\end{tabular}

(e)

\begin{tabular}{|c|c|c|c|c|}
\hline$\Psi_{0}$ & 0 & 0 & 0 & 0 \\
\hline 0 & $\Psi_{1}$ & 0 & 0 & 0 \\
\hline 0 & 0 & $\Psi_{2}$ & 0 & 0 \\
\hline 0 & 0 & 0 & $\cdots$ & 0 \\
\hline 0 & 0 & 0 & 0 & $\Psi_{n}$ \\
\hline
\end{tabular}

(g)

\begin{tabular}{|c|c|c|c|c|}
\hline 0 & 0 & $\Psi_{n}$ & 0 & 0 \\
\hline 0 & 0 & $\vdots$ & 0 & 0 \\
\hline 0 & 0 & $\Psi_{2}$ & 0 & 0 \\
\hline 0 & 0 & $\Psi_{1}$ & 0 & 0 \\
\hline 0 & 0 & $\Psi_{0}$ & 0 & 0 \\
\hline
\end{tabular}

(b)

\begin{tabular}{|c|c|c|c|c|}
\hline 0 & 0 & $\Psi_{0}$ & 0 & 0 \\
\hline 0 & 0 & $\Psi_{1}$ & 0 & 0 \\
\hline 0 & 0 & $\Psi_{2}$ & 0 & 0 \\
\hline 0 & 0 & $\vdots$ & 0 & 0 \\
\hline 0 & 0 & $\Psi_{n}$ & 0 & 0 \\
\hline
\end{tabular}

(d)

\begin{tabular}{|c|c|c|c|c|}
\hline$\Psi_{n}$ & 0 & 0 & 0 & 0 \\
\hline 0 & $\cdots$ & 0 & 0 & 0 \\
\hline 0 & 0 & $\Psi_{2}$ & 0 & 0 \\
\hline 0 & 0 & 0 & $\Psi_{1}$ & 0 \\
\hline 0 & 0 & 0 & 0 & $\Psi_{0}$ \\
\hline
\end{tabular}

(f)

\begin{tabular}{|c|c|c|c|c|}
\hline 0 & 0 & 0 & 0 & $\Psi_{0}$ \\
\hline 0 & 0 & 0 & $\Psi_{1}$ & 0 \\
\hline 0 & 0 & $\Psi_{2}$ & 0 & 0 \\
\hline 0 & $\cdots$ & 0 & 0 & 0 \\
\hline$\Psi_{n}$ & 0 & 0 & 0 & 0 \\
\hline
\end{tabular}

(h)

FIGURE 1: Fractional heat masks on eight directions: $0^{\circ}, 45^{\circ}, 90^{\circ}, 135^{\circ}, 180^{\circ}, 225^{\circ}, 270^{\circ}$, and $315^{\circ}$.

0.9 , using "Boat" corrupted by Gaussian noise with standard deviation $\sigma$ of 25. When the value of $\alpha$ is small, this leads to small value of PSNR of the denoised image, while for large value of $\alpha$, PSNR drops dramatically. Therefore, the trade-off between $\alpha$ and PSNR is required to remove noise. We should choose the optimal value of $\alpha=0.5$. Figure 3 shows the behavior of the PSNR for values of $\gamma$, varying from 0.1 to 1 , at $\alpha=0.5$. The trade-off between $\gamma$ and PSNR is chosen, $\gamma=0.9$. When the value of $\gamma$ is an integer number, this method results in lower value of PSNR. These values remain constant at 5.6 for all values of $\alpha$, as shown in Figure 4, while high value of PSNR is achieved when $\gamma$ is fractional number.
Figures 5 and 6 show the experimental results of all images. From the human visual system effect, we can conclude that the proposed FHD denoising algorithm has good denoising performance for all testing images.

The aim of this study is to ensure that our proposed algorithm removes noise effectively from the corrupted images by comparing our algorithm with two standard filters for image denoising, which are the standard Gaussian filter and Wiener filter.

Table 1 shows the numerical evidence of the efficiency of the proposed FHD algorithm with different values of $\sigma$ for two sets of standard images (grayscale and color 


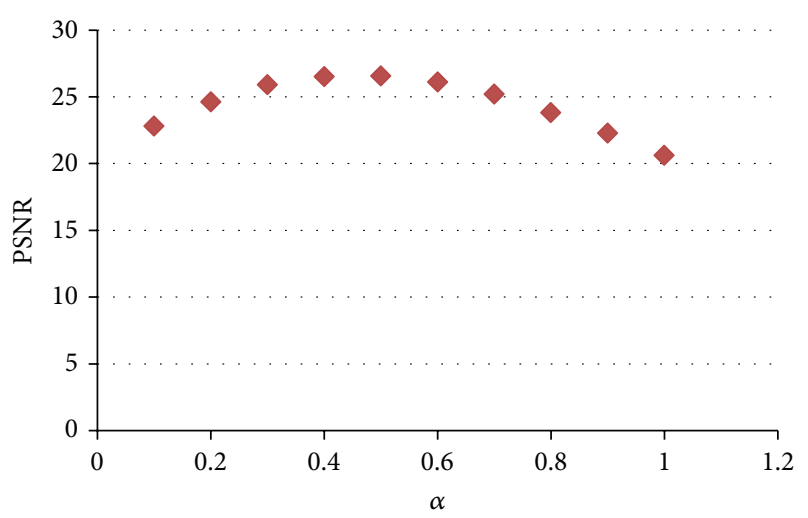

FIGURE 2: Choice of fractional $\alpha$ at $\gamma=0.9$, for "Boat" corrupted by Gaussian noise with $\sigma=25$.

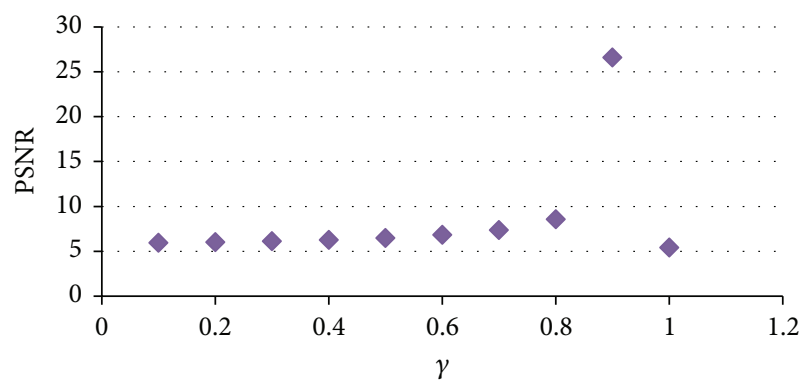

Figure 3: Choice of fractional $\gamma$ at $\alpha=0.5$, for "Boat" corrupted by Gaussian noise with $\sigma=25$.

images). The visual experiments illustrate that the proposed FHD algorithm achieves the maximum PSNR value over Gaussian smoothing filter and Wiener filter, although the PSNR rate is slightly higher than Gaussian filter and Wiener filter. The reason is due to the fact that the enhancing of the fractional operators only affects the pixel values that are changing sharply (high frequency of image), while no significant changes occur in the low frequency of image.

\section{Comparison with Other Methods}

To further verify the performance of our proposed algorithm, Table 2 shows the comparison of the experimental results of the proposed algorithm with other denoising algorithms for "Boat" image with the noise standard deviation $\sigma$ values of 15 , 20, and 25. All comparisons are made on the basis of PSNR.

To our knowledge, no previous studies have been done using FHD for image denoising. Therefore, we compare our study with those which employed other methods. For using linear heat equation, Cuesta et al. in [23] have developed a novel approach for image denoising, with time fractional differential equation, while in our proposed algorithm we used time-space fractional differential equation that utilizes the convex solution of FHD for image denoising.

$\mathrm{Hu}$ et al. in [10] proposed a novel fractional integral image denoising algorithm, based on fractional calculus Riemann-Liouville definition for image denoising. However,

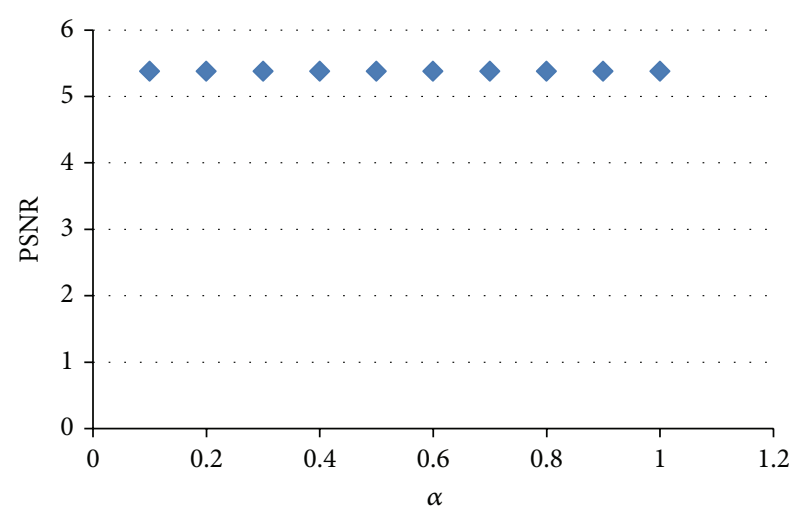

FIgURE 4: Choice of integer $\alpha$ at $\gamma=1$, for "Boat" corrupted by Gaussian noise with $\sigma=25$.

TABLE 1: PSNR of denoised images with different Gaussian noise values.

\begin{tabular}{|c|c|c|c|c|}
\hline PSNR & Noisy image & Gaussian smoothing & Wiener filter & FHD \\
\hline Image & & Grayscale "Lena" & & \\
\hline$\sigma=15$ & 24.65 & 28.25 & 29.87 & 31.72 \\
\hline$\sigma=20$ & 22.15 & 25.92 & 29.62 & 30.03 \\
\hline$\sigma=25$ & 20.25 & 24.02 & 27.65 & 28.59 \\
\hline Image & & Grayscale "Taj” & & \\
\hline$\sigma=15$ & 24.81 & 28.43 & 30.28 & 30.35 \\
\hline$\sigma=20$ & 22.35 & 26.01 & 28.78 & 29.04 \\
\hline$\sigma=25$ & 20.48 & 24.16 & 27.24 & 27.85 \\
\hline Image & & Grayscale "Boat" & & \\
\hline$\sigma=15$ & 24.67 & 28.36 & 30.77 & 31.20 \\
\hline$\sigma=20$ & 22.20 & 25.93 & 28.40 & 29.70 \\
\hline$\sigma=25$ & 20.30 & 24.03 & 27.56 & 28.40 \\
\hline Image & & Color "Pepper" & & \\
\hline$\sigma=15$ & 24.79 & 28.51 & 28.57 & 28.88 \\
\hline$\sigma=20$ & 22.36 & 26.08 & 27.72 & 27.99 \\
\hline$\sigma=25$ & 20.48 & 24.17 & 26.79 & 27.06 \\
\hline Image & & Color "House" & & \\
\hline$\sigma=15$ & 24.68 & 28.34 & 30.63 & 31.18 \\
\hline$\sigma=20$ & 22.21 & 25.92 & 29.98 & 29.73 \\
\hline$\sigma=25$ & 20.32 & 24.02 & 28.24 & 28.36 \\
\hline
\end{tabular}

[11] proposed an image denoising algorithm called generalized fractional integral filter based on generalized SrivastavaOwa fractional integral operator.

Table 2 provides an overall view of the performance of different methods, although these methods have used different images with different noise standard deviation $\sigma$ values. For testing images "Boat," the values of PSNR for FHD are slightly larger than of the three methods. The proposed algorithms for the image denoising effectively remove noise. The good PSNR of the proposed FHD algorithm acts as one of the important parameters in judging its performance. 

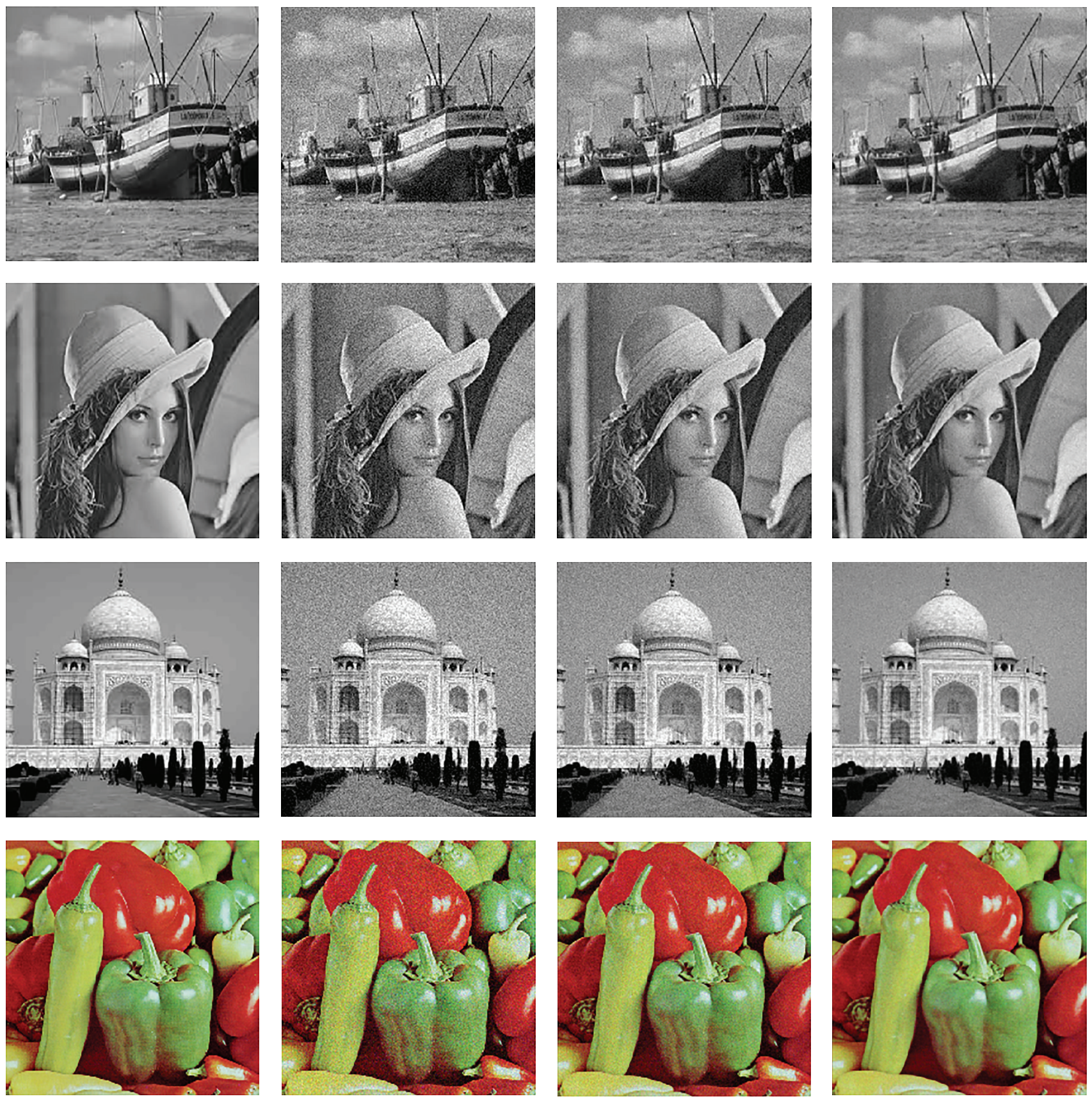

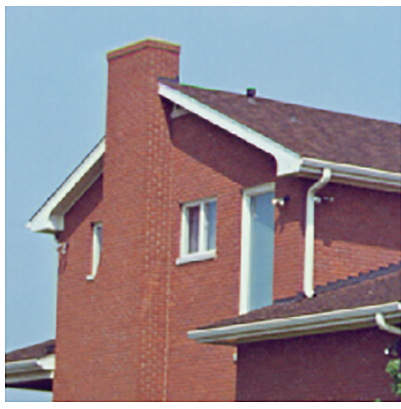

(a)

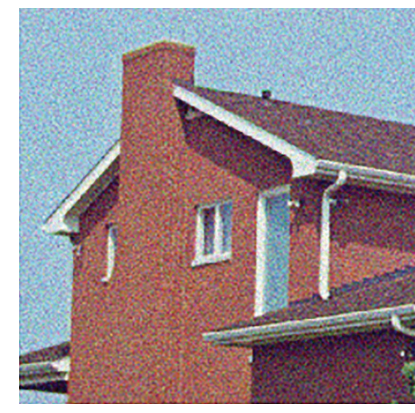

(b)

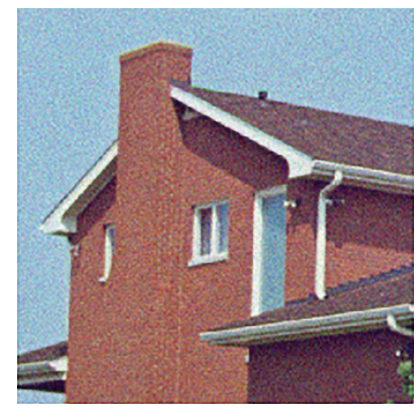

(c)

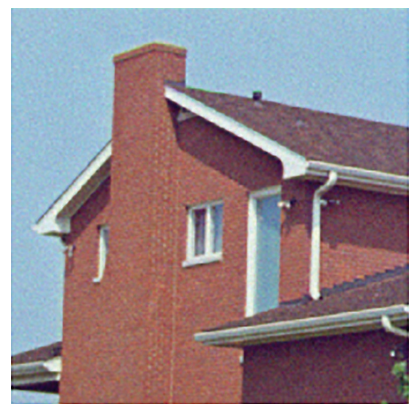

(d)

FIGURE 5: Denoising results, (a) original image, (b) corrupted image with Gaussian noise with $\sigma$ value of 15, (c) Gaussian smoothing filter, and (d) FHD filter. 

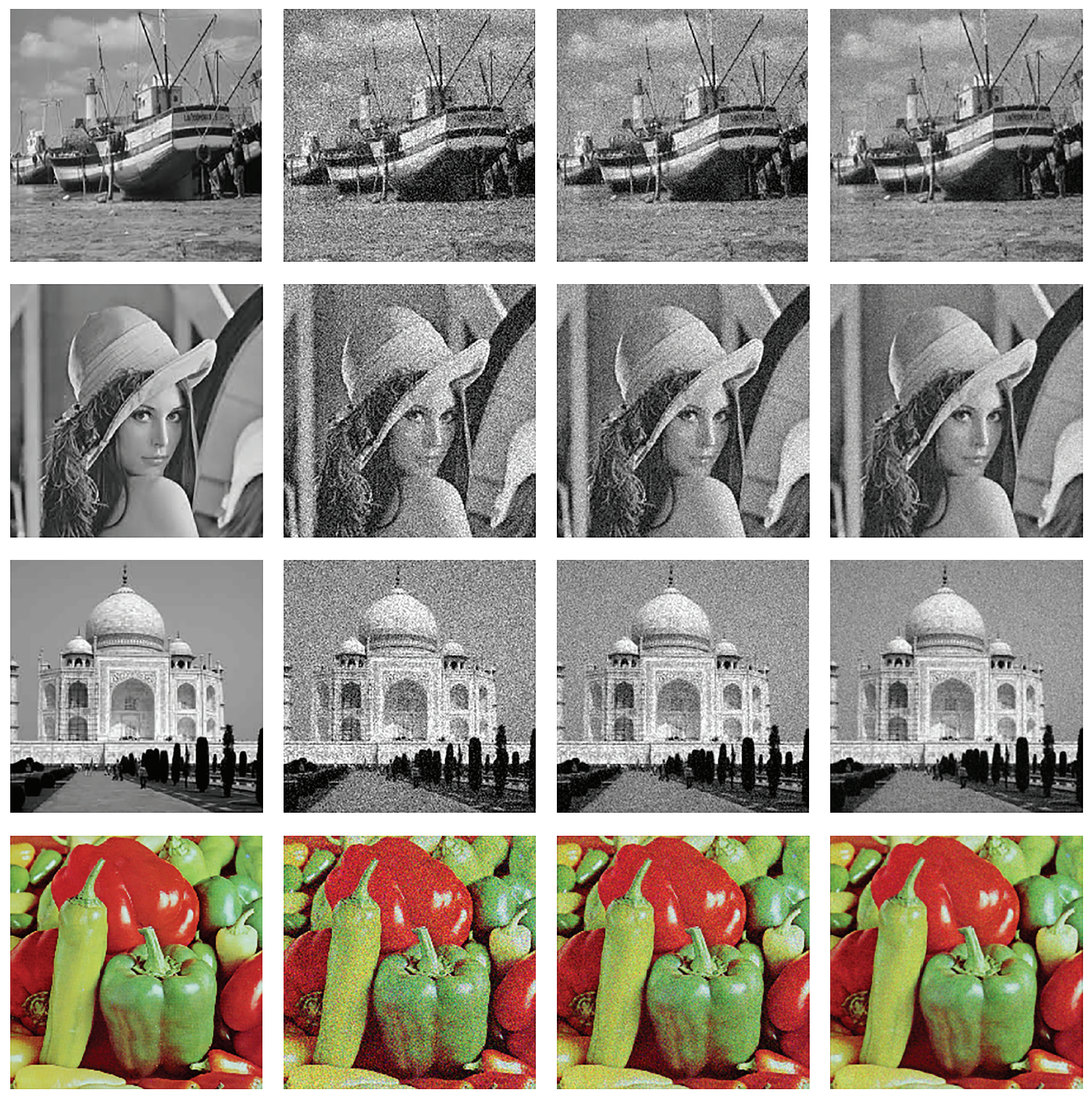

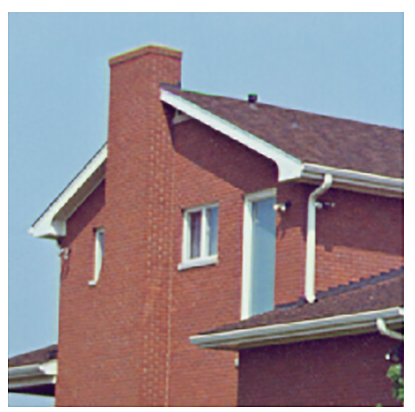

(a)

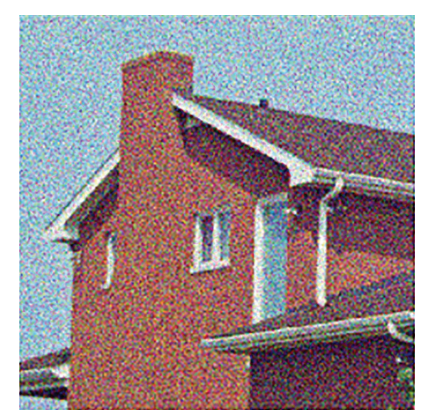

(b)

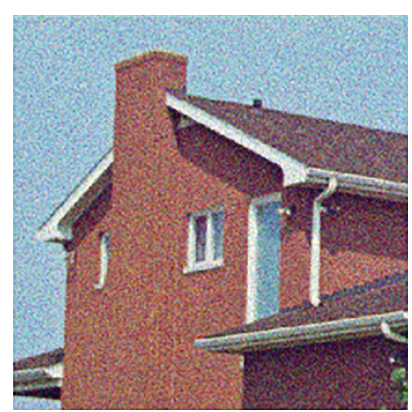

(c)

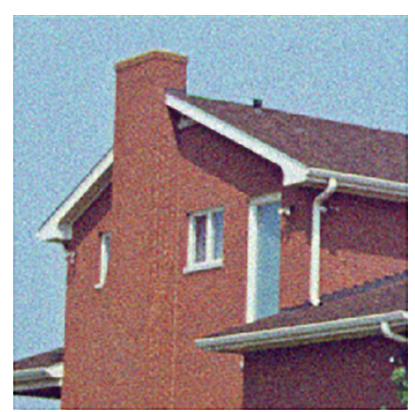

(d)

FIGURE 6: Denoising results, (a) original image, (b) corrupted image with additive white Gaussian noise with $\sigma$ value of 25, (c) Gaussian smoothing filter, and (d) FHD filter. 
TABLE 2: Comparison of the experimental results for the standard image "Boat" with other methods.

\begin{tabular}{lcccc}
\hline \multirow{2}{*}{ Noise standard deviation $\sigma$} & {$[23]$} & {$[10]$} & {$[11]$} & FHD filter PSNR (dB) \\
\hline 15 & PSNR $(\mathrm{dB})$ & PSNR $(\mathrm{dB})$ & PSNR $(\mathrm{dB})$ & 31.20 \\
20 & 14.88 & 29.20 & 29.93 & 29.70 \\
25 & 13.50 & 27.91 & 28.01 & 28.40 \\
\hline
\end{tabular}

\section{Conclusion}

A new image denoising filter based on convex solution of fractional heat equation with regularized fractional power parameters is introduced to remove Gaussian noise. Both visual perception and PSNR are used to evaluate the denoising performance of proposed FHD algorithm. Experiments demonstrate that the improvements achieved in PSNR are competent with the Gaussian smoothing filter and Wiener filter. We analyzed the influence of parameters $\alpha$ and $\gamma$ for images corrupted by Gaussian noise with standard deviation $\sigma$ value of 25 on the performance of PSNR. An additional interesting property of our proposed algorithms is the characteristic of the denoising filter that can be adjusted easily by changing the two values of $\alpha$ and $\gamma$ of the proposed masks. Future works involve extending the proposed method to be done for texture enhancement for digital images using fractional heat equation.

\section{Conflict of Interests}

The author declares that he has no conflict of interests.

\section{Acknowledgment}

This research has been funded by University of Malaya, by the HIR Project UM.C/625/1/HIR/132.

\section{References}

[1] I. Podlubny, Fractional Differential Equations, vol. 198 of Mathematics in Science and Engineering, Academic Press, San Diego, Calif, USA, 1999.

[2] J. Sabatier, O. P. Agrawal, and J. T. Machado, Advances in Fractional Calculus: Theoretical Developments and Applications in Physics and Engineering, Springer, New York, NY, USA, 2007.

[3] V. Lakshmikantham and S. Leela, "A Krasnoselskii-Kreintype uniqueness result for fractional differential equations," Nonlinear Analysis: Theory, Methods \& Applications, vol. 71, no. 7-8, pp. 3421-3424, 2009.

[4] A. A. Kilbas, H. M. Srivastava, and J. J. Trujillo, Preface, NorthHolland Mathematics Studies, 2006.

[5] H. M. Srivastava, M. Darus, and R. W. Ibrahim, "Classes of analytic functions with fractional powers defined by means of a certain linear operator," Integral Transforms and Special Functions, vol. 22, no. 1, pp. 17-28, 2011.

[6] D. Baleanu, J. A. T. Machado, and A. C. Luo, Fractional Dynamics and Control, Springer, New York, NY, USA, 2012.

[7] R. L. Magin, O. Abdullah, D. Baleanu, and X. J. Zhou, "Anomalous diffusion expressed through fractional order differential operators in the Bloch-Torrey equation," Journal of Magnetic Resonance, vol. 190, no. 2, pp. 255-270, 2008.

[8] H. A. Jalab and R. W. Ibrahim, "Texture enhancement for medical images based on fractional differential masks," Discrete Dynamics in Nature and Society, vol. 2013, Article ID 618536, 10 pages, 2013.

[9] H. A. Jalab and R. W. Ibrahim, “Texture feature extraction based on fractional mask convolution with cesáro means for contentbased image retrieval," in PRICAI 2012: Trends in Artificial Intelligence, pp. 170-179, Springer, New York, NY, USA, 2012.

[10] J. Hu, Y. Pu, and J. Zhou, "A novel image denoising algorithm based on riemann-liouville definition," Journal of Computers, vol. 6, no. 7, pp. 1332-1338, 2011.

[11] H. A. Jalab and R. W. Ibrahim, "Denoising algorithm based on generalized fractional integral operator with two parameters," Discrete Dynamics in Nature and Society, vol. 2012, Article ID 529849, 14 pages, 2012.

[12] J. $\mathrm{Hu}, \mathrm{Y} . \mathrm{Pu}$, and J. Zhou, "Fractional integral denoising algorithm and implementation of fractional integral filter," Journal of Computational Information Systems, vol. 7, no. 3, pp. 729-736, 2011.

[13] Y.-F. Pu, J.-L. Zhou, and X. Yuan, "Fractional differential mask: a fractional differential-based approach for multiscale texture enhancement," IEEE Transactions on Image Processing, vol. 19, no. 2, pp. 491-511, 2010.

[14] H. Guo, X. Li, C. Qing-Li, and W. Ming-Rong, "Image denoising using fractional integral," in Proceedings of the IEEE International Conference on Computer Science and Automation Engineering (CSAE '12), vol. 2, pp. 107-112, Zhangjiajie, China, May 2012.

[15] H. A. Jalab and R. W. Ibrahim, "Texture enhancement based on the Savitzky-Golay fractional differential operator," Mathematical Problems in Engineering, vol. 2013, Article ID 149289, 8 pages, 2013.

[16] J. L. Vazquez, "Barenblatt solutionsand asymptotic behaviour for a nonlinear fractional heat equation of porous medium type," http://arxiv.org/abs/1205.6332.

[17] I. Karatay and S. R. Bayramoglu, "A characteristic difference scheme for time-fractional heat equations based on the CrankNicholson difference schemes," Abstract and Applied Analysis, vol. 2012, Article ID 548292, 11 pages, 2012.

[18] A. H. Bhrawy, E. H. Doha, D. Baleanu, and S. S. Ezz-Eldien, "A spectral tau algorithm based on Jacobi operational matrix for numerical solution of time fractional diffusion-wave equations," Journal of Computational Physics, 2014.

[19] C. Lixia, "Improved de-noising algorithm on heat equation," in Proceedings of the 3rd International Conference on Genetic and Evolutionary Computing (WGEC '09), IEEE, Guilin, China, October 2009.

[20] K. Lakshmi, R. Parvathy, S. Soumya, and K. P. Soman, "Image denoising solutions using heat diffusion equation," in Proceedings of the International Conference on Power, Signals, Controls 
and Computation (EPSCICON '12), IEEE, Thrissur, China, January 2012.

[21] Y. Xiaojun and D. Baleanu, "Fractal heat conduction problem solved by local fractional variation iteration method," Thermal Science, vol. 17, no. 2, pp. 625-628, 2013.

[22] R. W. Ibrahim and H. A. Jalab, "Time-space fractional heat equation in the unit disk," Abstract and Applied Analysis, vol. 2013, Article ID 364042, 7 pages, 2013.

[23] E. Cuesta, M. Kirane, and S. A. Malik, "Image structure preserving denoising using generalized fractional time integrals," Signal Processing, vol. 92, no. 2, pp. 553-563, 2012. 


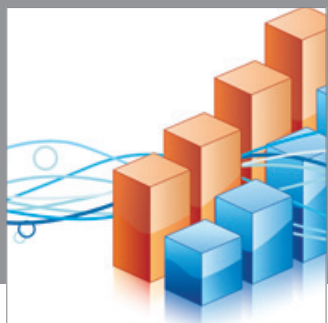

Advances in

Operations Research

mansans

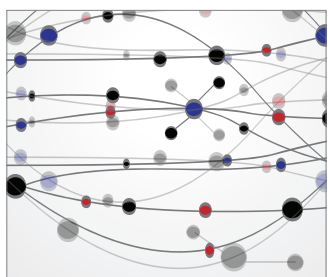

The Scientific World Journal

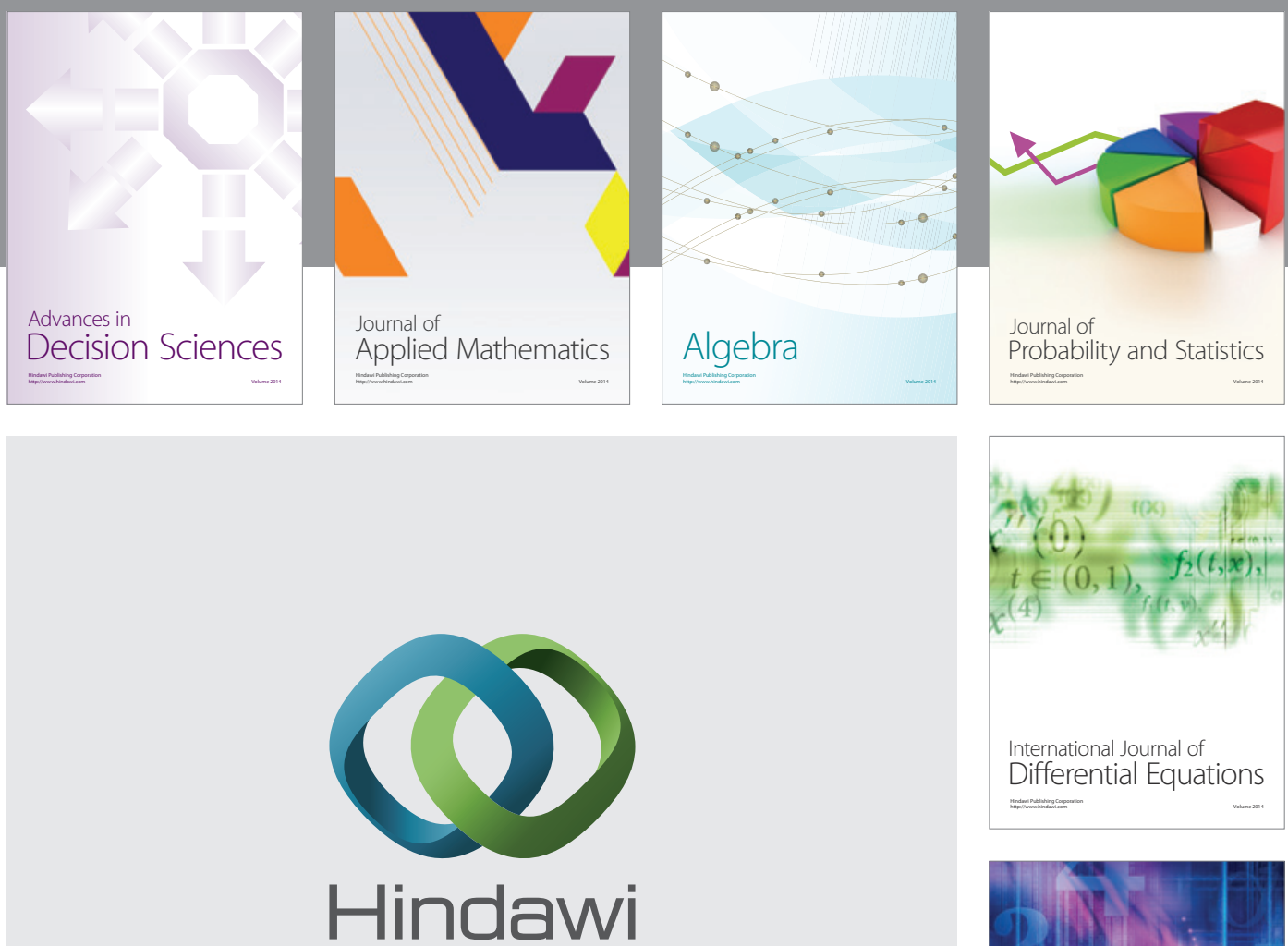

Submit your manuscripts at http://www.hindawi.com
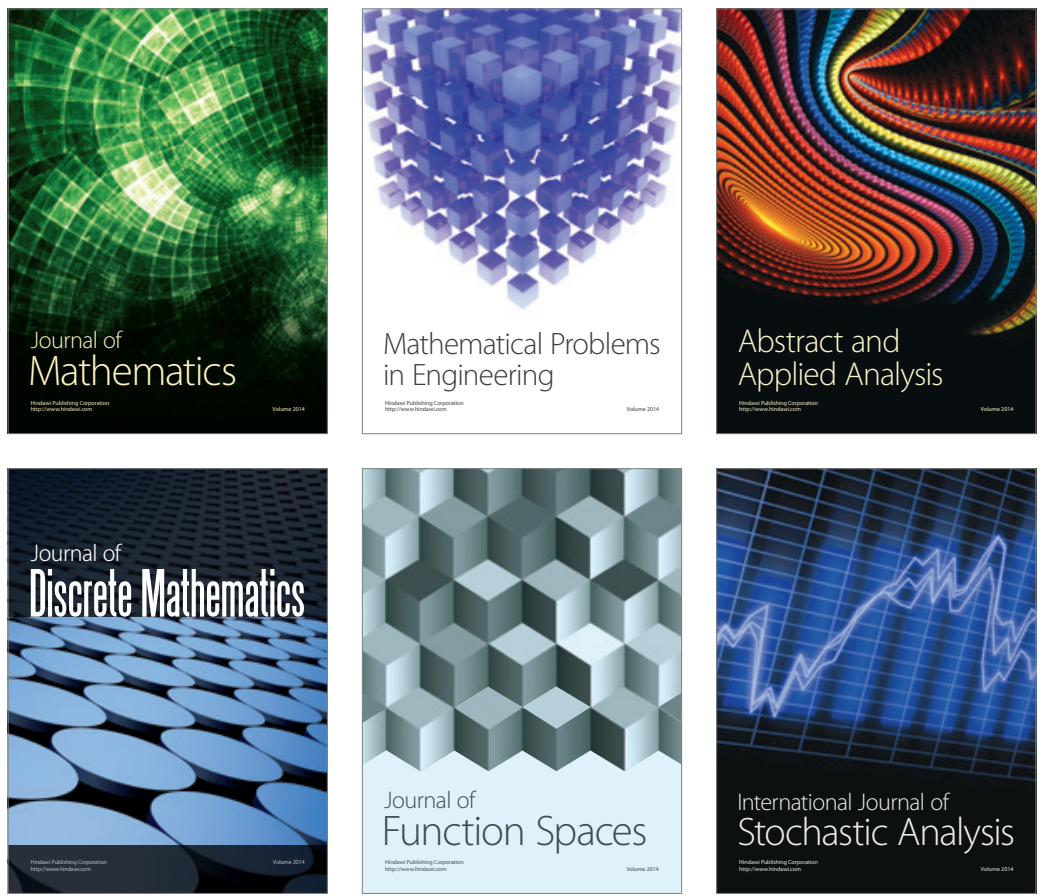

Journal of

Function Spaces

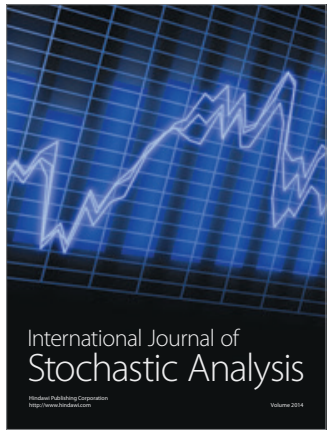

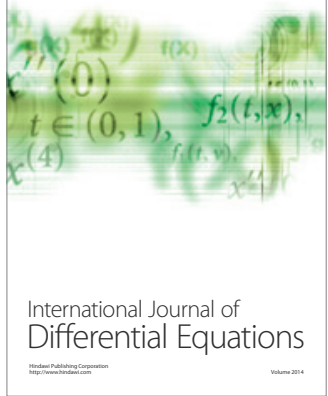
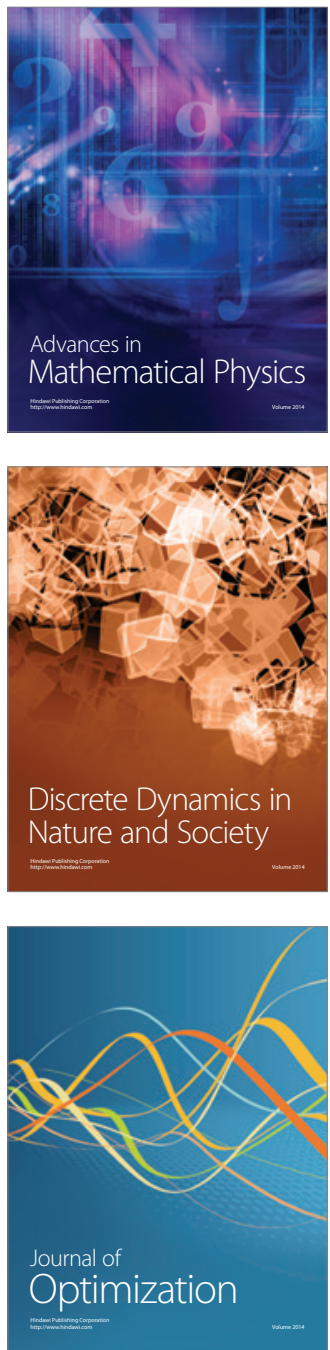